\title{
A SIMPLE EXPANSIBILITY TEST FOR DETERMINING THE WELDING PROPERTIES OF GLASSES ${ }^{1}$
}

\author{
BY DONAI, Fi. SHaRP
}

ABSTRACT

Simple method of determining the relative expansibilities of two glasses.Place two rods of glass side by side with the ends flush, heat these ends, weld together by pinching with forceps and then pull out a thread or thin strip with the two glasses forming opposite sides. When cool the thread will bend toward the glass having the higher total expansion up to the softening point of the softer glass. This test may be used for quantitative determination of coefficients of expansion if a series of glasses of known expansibilities are available for comparison. The method is of particular value for investigating the welding properties of glasses, for whether or not two glasses can safely be welded together may be determined directly from the amount of curvature of the thread.

In the factory manipulation of colored and clear glasses, it is frequently found necessary to weld together two glasses, or to coat a thin layer of colored glass with a thicker layer of clear glass. The former is usually called welding or casing, and is employed in the manufacture of ornamental and fancy wares. The latter is called flashing, and applies particularly to the manufacture of colors such as copper ruby where the depth of color is so great that the glass must be blown very thin in order to transmit an appreciable amount of light. It is consequently necessary in the latter case to coat the thin layer of colored glass with a much heavier layer of clear glass to give the ware the requisite mechanical strength and "body" to bear working in the blowing room and in its later use. The manufacturer of glass laboratory apparatus also, must often join tubing from two different sources, or seal some tubing to a piece of apparatus from another maker. It, therefore, becomes a matter of importance to learn whether or not the two glasses will seal without subsequent fracture.

It has been found that some glasses of widely different com-

${ }^{1}$ Received, July 17, 1920. 
position and physical properties can be welded together under certain circumstances and in certain shapes. The compound glass which has found some use for boiler gauge tubes is an excellent example of this kind of welding. These tubes are made by covering a layer of glass of one expansibility with a layer of glass of smaller expansibility. It is the difference in the expansion coefficients which in this case makes the compound gauge tube so tough.

In general, however, two glasses can not be welded or sealed together unless their coefficients of expansion are very nearly identical. In the compound tubes mentioned above it is possible to effect a seal, only because the strain is symmetrical and of such character that the forces brought into play in the weakest part of the tube are forces of compression rather than of tension.

Appert ${ }^{1}$ suggests that the conditions necessary for successful welding are: (a) The glasses must have the same coefficients of thermal expansion over the range of temperature to which the finished articles are likely to be submitted; $(b)$ the diathermancies, or power of transmitting heat, must be equal; $(c)$ the glasses must be inert and sustain no change whatever during the heating necessary in welding. He states also that the first condition is the most important and applies to all cases while the other two are only of secondary importance. A method of determining the state of tension existing between two glasses, which is described in detail in his article, might, indeed, find good application in the blowing room of a glass factory.

This method consists of blowing three bulbs from combinations of the two glasses under test. Calling the glasses A and B; one of the bulbs is blown of A glass alone and is reserved as the standard. The other two bulbs are made of both $A$ and $B$ glassesnumber 1 of a layer of $A$ coated with a layer of $B$, and number 2 of a layer of $B$ coated with $A$. The determination of the welding properties of the two glasses is then made by finding the resistance of numbers 1 and 2 to shock, as compared with the standard bulb of A glass. If the glasses differ in expansibility one of the bulbs is likely to burst violently while the other will be very resistant to shock due to the similarity to toughened or oil-

'Appert, Bull. Soc. d'encour. l'ind. nat., Jan., Feb., 1919. 
hardened glass. When the bulbs number 1 and 2 do not seem to differ from the standard in their susceptibility to breakage or to cutting with the diamond, the bulbs are perfectly sealed and no state of tension exists.

While this test is undoubtedly satisfactory for use in the blowing room of a glass factory where the glass may be had in quanticy, and may be manipulated on the blowing iron, it is not suitable for laboratory use, nor is it convenient for the lampworker's use before the blast flame. A method that has been used by the author for several years, and found very satisfactory, permi:s a test in the laboratory by means of a blast lamp, and is both quick and reliable. It has also the advantage of requiring only very small pieces of the glass under test.

The two glasses, whose properties of sealing together are to be tested, are worked out before the blast lamp or over a Meker burner into small rods. The ends of these rods are then heated

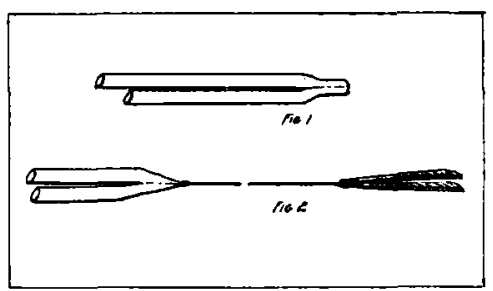

to softness and the two rods held together, and along side of one another, so that the ends which were softened weld together and form a single rod (Fig. 1). The junction is now carefully heated to softness and shaped slightly with a pair of forceps, into a form which may easily be pulled out. The end of the junction is now seized with the forceps in order to support it, and the flame allowed to strike on the thinner area of the compound strip that has been formed, in such a manner as to heat both glasses equally. As soon as the junction is soft enough, the rods are removed from the flame, still being held with the forceps, and the junction pulled out quickly into a thin strip or thread about 10-15 inches long, the tension being maintained until the softened glass has cooled below its deformation temperature (Fig. 2). 'The heavy end formerly held in the forceps. 
is now broken off and the thread held in a vertical position for examination.

The thin thread formed by drawing out the junction of the two pieces of cane is in reality a strip similar to the familiar metal thermostat. If the glasses composing the strip have different coefficients of expansion, a greater contraction occurs on one side of the strip than on the other and the strip bends into a curve, the glass of higher coefficient of expansion being on the inside of the curve. Glasses differing greatly in expansibility will give strips that will actually wind up into spirals when the tension is released, while those differing but little will show only a very slight departure from straightness. No curvature at all would, of course, be an indication that the glasses had identical expansion coefficients.

In employing this test as a criterion for satisfactory welding, it has been found that a very slight curvature, that is, a small difference in expansibility, indicates that the glasses will weld together without subsequent fracture, if well annealed. If the strip is absolutely straight there is no doubt that the two glasses may be used for any type of cased ware or for blowing even the most delicate apparatus before the blast lamp. A curvature of any appreciable amount, however, means that a satisfactory product can not be made of the glass combination.

Although the most frequent use of a test of this sort would doubtless be to determine whether or not two glasses had the same expansibility without regard for the amount of this expansibility, still the method can be made a quantitative one in a very simple manner.

A number of glass rods of small diameter and known expansion coefficients are obtained, ranging in value from 0.0000030 to 0.000011 by steps of about five in the seventh decimal place. This requires about sixteen or more different glasses. A suitable rack or tray should be provided and properly labeled so that easy access may be had to each variety.

If it is desired to determine the expansion coefficient of a new glass, a sealing test such as has been described above is made on the sample, using as a standard the one of the glasses of known expansibility which the experimental glass is judged to be most 
nearly like. From the result of this test the operator can easily tell in which direction the expansibility of the new glass lies, and a different standard is tested against it, until two known samples are found, one of higher and one of lower expansibility than the unknown. It now becomes easy to estimate within one or two units in the seventh decimal place, what is the coefficient of the unknown glass. By pulling the strip out rapidly into a fine thread the delicacy of the test is much increased and the method becomes very accurate.

The use of this method involves neither delicate apparatus nor a skilled operator, but may be used by a novice with almost no experience with a blast lamp. The standards must of course, be obtained by direct measurement or from a factory in which the expansion coefficients of the glasses have been accurately determined by careful laboratory methods.

In employing this test for factory control and laboratory experimentation in the several years it has been in use by the author, he has never been disappointed by obtaining misleading results. Several times actual measurements have been made to confirm the values arrived at by this method and in no case have the two methods failed to agree.

In conclusion, the author would like to express his thanks to Dr. J. T. L,ittleton of the Corning Glass Works, who very kindly permitted the publication of this article.

HAMBURG, $\mathbf{N}$. $\mathbf{Y}$. 